\title{
Efeito da temperatura e do regime de luz na germinação de sementes de Palmeira-leque-da-China (Livistona chinensis (Jack.) R. Br. ex. Mart.) ${ }^{(1)}$
}

\author{
NILCE NAOMI KOBORI(2), KATHIA FERNANDES LOPES PIVETTA ${ }^{(3)}$, MARIA ESMERALDA SOARES PAYÃO DEMATTÊ(4), \\ BRENO MARQUES DA SILVA E SILVA ${ }^{(5)}$, PETTERSON BAPTISTA DA LUZ ${ }^{(5)}$ e RICARDO SOARES PIMENTA ${ }^{(5)}$
}

\begin{abstract}
RESUMO
A Palmeira-leque-da-China é uma espécie bastante ornamental e muito utilizada em paisagismo. O objetivo deste trabalho foi verificar a influência de diferentes temperaturas e regimes de luz na germinação de sementes desta espécie. O delineamento experimental adotado foi inteiramente casualizado, em esquema fatorial $6 \times 2$ (seis temperaturas - constantes de 20, 25, 30, $35^{\circ} \mathrm{C}$ e alternadas de $20-30$ e $25-35^{\circ} \mathrm{C}$ - associadas a dois regimes de luz -8 horas de luz ou escuro contínuo). O experimento foi realizado em câmara de germinação do tipo BOD (Biochemical Oxygen Demand), com quatro repetições de 25 sementes, em caixas gerbox transparentes ou pretas, contendo vermiculita. As avaliações foram realizadas diariamente, considerando-se germinadas as sementes que apresentavam a protrusão do botão germinativo. Foi constatada alta porcentagem de germinação - 96 a 99\% - independentemente da temperatura e do regime de luz, e também maior índice de velocidade de germinação na temperatura alternada de $25-35^{\circ} \mathrm{C}$, com regime de 8 horas de luz.

Palavras-chave: palmeira, diásporo, botão germinativo, índice de velocidade de germinação.
\end{abstract}

\section{ABSTRACT \\ Effects of temperature and light regime on seed germination of Chinese fan palm (Livistona chinensis (Jack.) R. Br. ex. Mart.)}

\begin{abstract}
Chinese fan palm is an ornamental species very appreciated for landscaping. The objective of this research was to study the influence of different temperatures and light regimes in this species seed germination. The experiment was conducted under a complete randomized experimental design in a factorial arrangement $6 \times 2$ (six temperatures: constant of $20^{\circ} \mathrm{C}, 25^{\circ} \mathrm{C}, 30^{\circ} \mathrm{C}, 35^{\circ} \mathrm{C}$ and alternated of $20-30^{\circ} \mathrm{C}$ e $25-35^{\circ} \mathrm{C}$; associated to two light regimes: 8 hours of light or continuous dark). The experiment was conducted in germination chambers, in four replications of 25 seeds, in transparent or dark plastic boxes containing vermiculite. Evaluation was realized daily and seeds were considered germinated when presented the germinative button protrusion. Seeds of this species had high germination percentage (96 to 99\%), independently of temperature and light regime, and higher germination velocity index was observed in the alternate temperature of $25-35^{\circ} \mathrm{C}$, with 8 hours of light.
\end{abstract}

Keywords: palm, diaspore, germinative button, germination velocity index.

\section{INTRODUÇÃO}

Livistona chinensis (Jack.) R. Br. ex. Mart., também conhecida como Palmeira-leque-da-China e falsa-latânia, embora originária do continente asiático, está amplamente distribuída nas Américas (MEEROW, 1991a), muito difundida no Brasil em parques, jardins e em vasos, quando jovem (LORENZI et al., 2004). É uma espécie considerada rústica, presente em regiões tropicais, de clima subtropical e temperado de todo o mundo, e desenvolve-se bem, tanto a pleno sol como a meia sombra, além de crescer em vários tipos de solos (GILMAN \& WATSON, 1993).

A germinação de sementes de palmeiras, de modo geral, é considerada lenta, desuniforme e, frequentemente, em baixa porcentagem. A propagação é feita quase que exclusivamente por meio de sementes, com grande variação no processo germinativo, influenciado por diversos fatores como o grau de maturação, a presença ou não do pericarpo, o tempo entre a colheita e a semeadura, a temperatura do ambiente, o substrato, entre outros (MEEROW, 1991b; BROSCHAT, 1994). A Família Arecaceae constitui um grupo especial do ponto de vista do melhoramento de plantas perenes, especialmente em função das dificuldades inerentes à reprodução vegetativa dos indivíduos, fato que dificulta, ou mesmo impossibilita o pleno uso dos genótipos superiores. Isso tem impacto direto nas estratégias de melhoramento e de produção de propágulos melhorados dessas espécies (KALIL FILHO \& RESENDE, 2001).

A temperatura e a luz são os principais fatores ambientais que afetam a germinação de sementes no solo, desde que haja disponibilidade de água e oxigênio. Para muitas espécies vegetais, quando são fornecidas condições

\footnotetext{
(1) Parte da Dissertação de Mestrado da primeira autora apresentada à UNESP - Faculdade de Ciências Agrárias e Veterinárias (FCAV), Campus de Jaboticabal. Bolsista CAPES. Recebido para publicação em 01/10/2007 e aceito em 24/03/2009

(2) Eng ${ }^{\mathrm{a}}$. Agrônoma, Mestre pelo Programa de Pós-graduação em Produção e Tecnologia de Sementes da FCAV - UNESP, Campus Jaboticabal, e Doutoranda em Fitotecnia - USP/ESALQ. E-mail: nnkobori@esalq.usp.br

(3) Prof ${ }^{\mathrm{a}}$. Dr ${ }^{\mathrm{a}}$. Assistente Depto. de Produção Vegetal da FCAV - UNESP, Via de Acesso Prof. Paulo Donato Castellane, s/n. 14884-900, Jaboticabal (SP), Brasil.kathia@fcav.unesp.br

(4) Prof ${ }^{\mathrm{a}}$. Dr ${ }^{\mathrm{a}}$. Adjunta Aposentada Voluntária Depto. de Produção Vegetal da FCAV - UNESP, Jaboticabal

(5) Pós-graduandos do Programa de Pós-graduação em Produção e Tecnologia de Sementes da FCAV - UNESP.
} 
adequadas de luz e umidade, a temperatura predominante determina não só a fração de sementes que germina como também a velocidade de germinação (ANDRADE, 1995).

Para alguns grupos ecológicos, a presença de luz pode influenciar a germinação de sementes (CASTRO \& HILHORST, 2004). Quanto às espécies sensíveis à luz, há sementes que germinam somente após rápida exposição à luz, outras que necessitam de período amplo de exposição, outras em que a germinação é desencadeada somente no escuro (VIDAVER, 1980) e sementes totalmente indiferentes à luz (VÁZQUEZ-YANES \& OROZCO-SEGOVIA, 1991).

Segundo VIDAVER (1980), BRYANT (1985) e VÁZQUEZ-YANES \& OROZCO-SEGOVIA (1990), o fitocromo é o pigmento receptor responsável pela captação de sinais luminosos, que podem ou não desencadear a germinação das sementes. O modo de ação desse pigmento depende do tipo de radiação incidente, pois luz com alta relação vermelho/vermelho-extremo (V/VE) pode induzi-lo a assumir a forma ativa (FVe), promovendo a germinação de sementes fotossensíveis; já luz com baixa relação V/VE pode levá-lo a assumir a forma inativa (FV), impedido a germinação.

De acordo com BROSCHAT (1994), é desconhecida a dependência de luz para a germinação de sementes de palmeiras. Para a espécie Rhapis excelsa, AGUIAR et al. (2005) verificaram não haver necessidade de luz para germinação à temperatura de $25^{\circ} \mathrm{C}$. SILVA et al. (1999) avaliaram a germinação de sementes de Aiphanes aculeata a partir de frutos em diferentes estádios de maturação, concluindo que a germinação foi melhor em frutos vermelhos, independentemente dos regimes de luz. Em função da resposta à luz, recomenda-se a utilização de luz branca (que contém os comprimentos de onda necessários para ativar ou desativar o fitocromo) para a germinação das sementes de diversas espécies (ZAIDAN \& BARBEDO, 2004).

Estudos da germinação são importantes para fornecer não só informações úteis à tecnologia de sementes, mas também para a compreensão da ecofisiologia das espécies vegetais (BORGHETTI \& FERREIRA 2004). O teste de germinação para avaliar a qualidade da semente, feito em laboratório, é realizado sob condições de temperatura e substrato ideais para cada espécie. De acordo com BEWLEY \& BLACK (1996), a temperatura influencia a porcentagem final e a velocidade de germinação, dentro de amplitudes térmicas definidas para cada espécie, denominadas máxima, ótima e mínima. O efeito da temperatura na germinação afeta a velocidade de absorção de água pelas sementes e pode alterar, entre outros aspectos, a porcentagem total, a velocidade e a uniformidade de germinação (CARVALHO \& NAKAGAWA, 2000; CASTRO \& HILHORST, 2004).

As temperaturas favoráveis à germinação de sementes de palmeiras variam de acordo com as espécies, sendo aceitáveis entre 20 e $40^{\circ} \mathrm{C}$, mas com melhores resultados entre 30 e $35^{\circ} \mathrm{C}$ para a maioria das espécies (MEEROW, 1991b). Sementes de muitas espécies da família Arecaceae, segundo BROSCHAT (1994), germinam melhor na faixa de 25 a $35^{\circ} \mathrm{C}$, enquanto LORENZI et al. (2004) consideraram favoráveis temperaturas entre 24 e $28^{\circ} \mathrm{C}$, com umidade relativa do ar de aproximadamente $70 \%$. Para Livistona rotundifolia, a temperatura alternada de $25-35^{\circ} \mathrm{C}$ proporcionou maior índice de velocidade de germinação (VIANA, 2003).

A padronização de métodos de análises visa à obtenção de resultados uniformes para um lote de sementes, analisado em diferentes laboratórios, por meio de procedimentos recomendados nas Regras para Análise de Sementes (BRASIL, 1992). No entanto, para espécies nativas e exóticas de menor interesse econômico, essa padronização é bastante escassa, representando menos de $0,1 \%$ das prescrições e recomendações. Os procedimentos são, então, estabelecidos por meio de experimentação prévia, que possibilite a avaliação da qualidade da semente. Nas últimas décadas, entretanto, houve um grande aumento de pesquisas na área de sementes florestais, devido ao crescente interesse econômico e conservacionista (OLIVEIRA et al., 1989).

Considerando-se a importância da propagação sexuada e a necessidade de fornecer subsídios para a tecnologia de sementes e preservação da espécie, o presente estudo teve como objetivo estudar o comportamento germinativo das sementes de Livistona chinensis (Jack.) R. Br. ex. Mart., visando determinar a melhor temperatura e os efeitos da luz e do escuro no processo.

\section{MATERIAL E MÉTODOS}

Os experimentos foram conduzidos no Laboratório de Sementes Hortícolas e Florestais do Departamento de Produção Vegetal da FCAV (Faculdade de Ciências Agrárias e Veterinárias) - Horticultura - UNESP.

Os cachos de Livistona chinensis (Jack.) R. Br. ex. Mart. foram colhidos no dia 13 de julho de 2005, de dez matrizes com aproximadamente 30 anos de idade, existentes na Coleção de Palmeiras da UNESP - Faculdade de Ciências Agrárias e Veterinárias (FCAV), Campus de Jaboticabal. Os frutos, maduros fisiologicamente, apresentavam coloração verde-azulada.

Após a colheita, esses frutos foram homogeneizados e mantidos imersos em água em temperatura ambiente, por 24 horas. O despolpamento consistiu na remoção do epicarpo e do mesocarpo dos frutos, por meio de atrito manual contra uma peneira. Os diásporos (sementes com o endocarpo aderido) foram enxaguados em água corrente e secos à sombra durante 24 horas. Antes do início dos tratamentos, os frutos foram selecionados visualmente, retirando-se aqueles mal-formados ou danificados. Para a determinação do teor de água, foram retiradas cinco amostras de 20 diásporos e, a partir do método da estufa a $105^{\circ} \mathrm{C} \pm 3^{\circ} \mathrm{C}$ por 24 horas (Brasil, 1992), verificou-se que foi de 19,3\%.

O delineamento experimental foi inteiramente casualizado, conduzido em esquema fatorial $6 \times 2$, ou seja, seis condições de temperaturas em BOD - Biochemical Oxygen Demand (constantes de $20,25,30,35^{\circ} \mathrm{C}$ e alternadas de $20-30^{\circ} \mathrm{C}$ e $25-35^{\circ} \mathrm{C}$, com $70 \%$ de umidade relativa do ar), associadas a dois regimes de luz ( 8 horas de luz ou escuro contínuo), com quatro repetições de 25 sementes. Os diásporos foram colocados em caixas gerbox desinfetadas com álcool, contendo como substrato vermiculita média. As regas foram realizadas quando necessário, a fim de manter a umidade do substrato. Para os tratamentos com luz, utilizou-se caixa plástica transparente $(11 \times 11 \times 3 \mathrm{~cm})$ e 
fotoperíodo de 8 horas; para os tratamentos no escuro contínuo, utilizou-se caixa plástica preta $(11 \times 11 \times 3 \mathrm{~cm})$, sendo a avaliação realizada em câmara escura, sob luz verde de segurança. $\mathrm{O}$ critério de germinação adotado para a avaliação foi o início da protrusão do botão germinativo. As observações para contagem de sementes germinadas em laboratório foram realizadas diariamente, até a estabilização do processo.

Foram calculados a porcentagem de germinação e o índice de velocidade de germinação (IVG), este último seguindo o critério estabelecido por MAGUIRE (1962). Os dados de porcentagem de germinação foram transformados em arc seno (x/100) 1/2, e as médias comparadas pelo teste de Tukey, a 5\% de probabilidade (BANZATTO \& KRONKA, 2006).

\section{RESULTADOS E DISCUSSÃO}

A germinação teve início a partir do quarto dia após a instalação dos tratamentos, com a emissão do botão germinativo, independentemente da luz, porém, dependendo da temperatura. Provavelmente, essas sementes foram colhidas já no estádio de maturidade fisiológica e, após o beneficiamento (despolpa), nas condições adequadas de água e temperatura, iniciaram o processo germinativo.

Nenhuma significância foi observada para a porcentagem de germinação nas diferentes temperaturas e nos regimes de luz analisados, bem como para as interações entre os fatores, constatando-se altas porcentagens de germinação, de 96 a 99\%. Por outro lado, a diferença no índice de velocidade de germinação (IVG) foi significativa para os dois fatores avaliados (tabela 1 e figuras 1 e 2): na temperatura de $20^{\circ} \mathrm{C}$, observou-se menor média de IVG; nas tempe- raturas de $25,30,35^{\circ} \mathrm{C}$ e na variação entre $20-30^{\circ} \mathrm{C}$, o IVG não diferiu estatisticamente; por outro lado, na temperatura alternada de $25-35^{\circ} \mathrm{C}$ e na presença de luz constatou-se maior velocidade de germinação.

Esses resultados assemelham-se aos de outros autores, que relataram temperaturas entre 20 e $40^{\circ} \mathrm{C}$ como aceitáveis para a germinação de sementes de palmeiras (MEEROW, 1991b), com melhores resultados entre 30 e $35^{\circ} \mathrm{C}$ para a maioria das espécies (BROSCHAT, 1994). Segundo estudo de CARPENTER (1988), a temperatura de $35^{\circ} \mathrm{C}$ promoveu maior germinação para quatro espécies de palmeiras estudadas, sendo que, as temperaturas acima ou abaixo de $35^{\circ} \mathrm{C}$ em 5 a $10^{\circ} \mathrm{C}$, normalmente retardaram e reduziram a germinação, tornando-a irregular e desuniforme para as espécies Acoelorraphe wrightii (Griseb \& H. Wendl). H. Wendl. ex. Becc., Coccothrinax argentata (N. J. Jacquin) L. H. Bailey, Sabal etonia Swingle ex. Nash. e Thrinax morrisii $\mathrm{H}$. Wendl. Essa mesma temperatura $\left(35^{\circ} \mathrm{C}\right)$ também foi a que proporcionou os melhores resultados de germinação de sementes para Thrinax parviflora Swartz (Pivetta et al., 2005a) e para Roystonea regia (Kunth) O.F. Cook (PENARIOL, 2005). Para outras espécies, como babaçu, tamareira-anã (IOSSI et al., 2003), pupunha (VILLALOBOS \& HERRERA, 1991) e piaçaveira (MELO, 2001), a temperatura mais adequada à maioria foi de $30^{\circ} \mathrm{C}$, enquanto temperaturas de $35^{\circ} \mathrm{C}$ ou superiores prejudicaram a germinação (YOCUM, 1961; REES, 1963; PINTO, 1971; ELLIS et al., 1985; CARPENTER, 1988; IOSSI et al., 2003).

Os resultados deste trabalho são semelhantes aos obtidos por CHATTY \& TISSAOUI (1999), em que, estudando-se as temperaturas de $5,15,25,35$ e $45^{\circ} \mathrm{C}$, as que apresentaram maior porcentagem de germinação de sementes de Livistona chinensis foram de $25^{\circ} \mathrm{C}$ e $35^{\circ} \mathrm{C}$.

Tabela 1. Médias obtidas para porcentagem de germinação e índice de velocidade de germinação de sementes de Livistona chinensis, submetidas a dois regimes de luz e seis temperaturas. Jaboticabal, SP, 2006.

Table 1. Means for germination percentage and germination velocity index of Livistona chinensis seeds, submitted to two light regimes and six temperatures. Jaboticabal, SP, 2006.

\begin{tabular}{|c|c|c|}
\hline Médias & Germinação (\%) $)^{1,2}$ & Índice de velocidade de germinação ${ }^{2}$ \\
\hline Luz & $81,7^{1}(97,9 \pm 1,5)^{2} \mathrm{~A}$ & $1,8800( \pm 0,18) \mathrm{A}$ \\
\hline Escuro & $80,9(97,5 \pm 1,75) \mathrm{A}$ & $1,5960( \pm 0,20) \mathrm{B}$ \\
\hline DMS & 5,5 & 0,1499 \\
\hline $20^{\circ} \mathrm{C}$ & $78,6(96,1 \pm 2,21) \mathrm{A}$ & $0,7894( \pm 0,08) \mathrm{C}$ \\
\hline $25^{\circ} \mathrm{C}$ & $81,9(98,0 \pm 1,99) \mathrm{A}$ & $1,6978( \pm 0,12) \mathrm{B}$ \\
\hline $30^{\circ} \mathrm{C}$ & $78,9(96,3 \pm 2,08) \mathrm{A}$ & $1,9011( \pm 0,03) \mathrm{B}$ \\
\hline $35^{\circ} \mathrm{C}$ & $81,7(97,9 \pm 1,59) \mathrm{A}$ & $1,7130( \pm 0,13) \mathrm{B}$ \\
\hline $20-30^{\circ} \mathrm{C}$ & $81,4(97,8 \pm 1,75) \mathrm{A}$ & $1,9035( \pm 0,09) \mathrm{B}$ \\
\hline $25-35^{\circ} \mathrm{C}$ & $85,1 \quad(99,3 \pm 1,03) \mathrm{A}$ & $2,4232( \pm 0,04) \mathrm{A}$ \\
\hline DMS & 14,1 & 0,3847 \\
\hline
\end{tabular}

${ }^{2}$ Dados não transformados \pm EPM (erro padrão da média); Médias seguidas da mesma letra na coluna não diferem entre si pelo Teste de Tukey a $5 \%$ de probabilidade $(\mathrm{P}>0,05)$; DMS: diferença mínima significativa. 


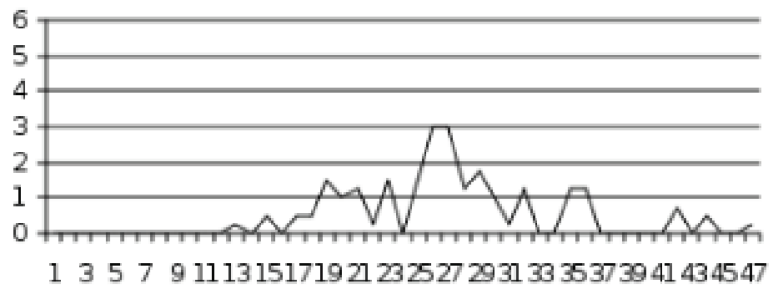

(A)

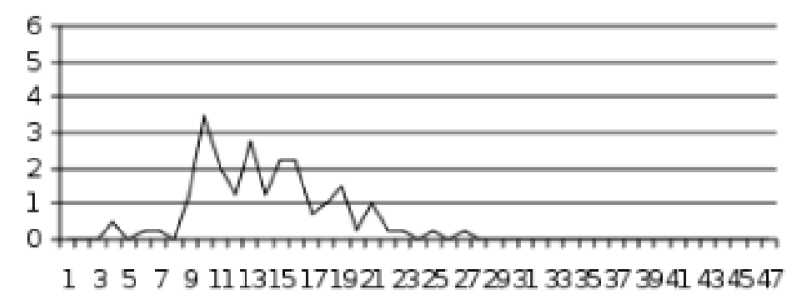

(C)

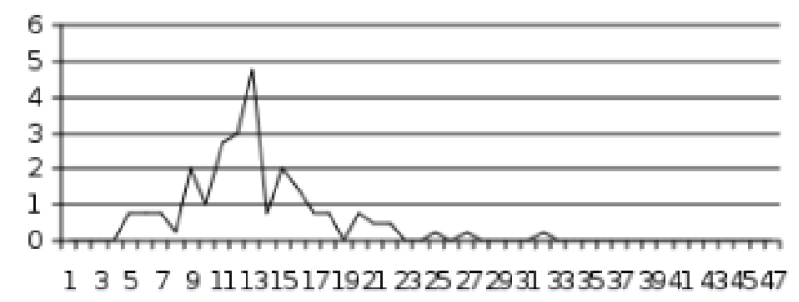

(E)

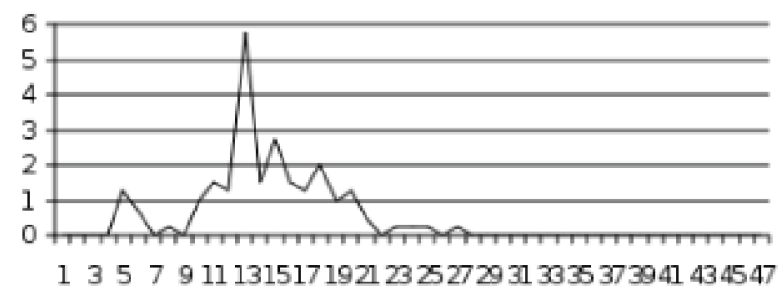

(B)

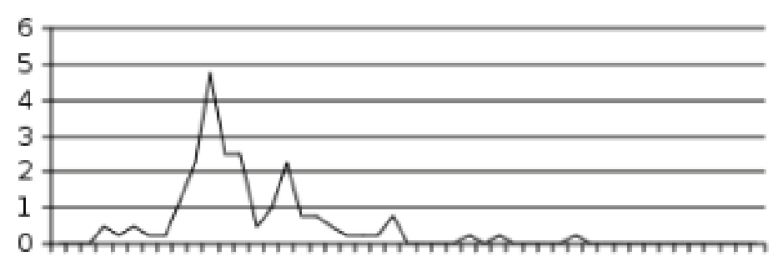

$1 \quad 357 \quad 911131517192123252729313335373941434547$

(D)

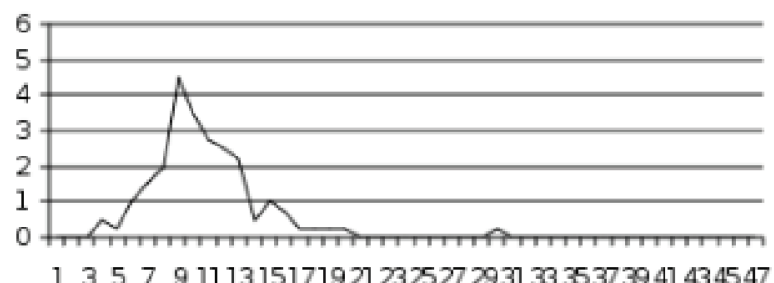

(F)

Figura 1. Número de sementes germinadas (eixo $\mathrm{Y}$ ) por dia (eixo $\mathrm{X}$ ) nas temperaturas de $20^{\circ} \mathrm{C}(\mathrm{A}), 25^{\circ} \mathrm{C}(\mathrm{B}), 30^{\circ} \mathrm{C}(\mathrm{C})$, $35^{\circ} \mathrm{C}(\mathrm{D}), 20-30^{\circ} \mathrm{C}(\mathrm{E}), 25-35^{\circ} \mathrm{C}(\mathrm{F})$ em regime de luz.

Figure 1. Number of germinated seeds (Y axis) per Day (X axis) at temperatures of $20^{\circ} \mathrm{C}(\mathrm{A}), 25^{\circ} \mathrm{C}(\mathrm{B}), 30^{\circ} \mathrm{C}(\mathrm{C}), 35^{\circ} \mathrm{C}$ (D), 20-30 $0^{\circ} \mathrm{C}$ ), $25-35^{\circ} \mathrm{C}(\mathrm{F})$ with light regime.

Em relação à luminosidade, não houve necessidade de luz para a germinação de sementes de Palmeira-leque-daChina, assim como para Rhapis excelsa (AGUIAR et al., 2005) e Aiphanes aculeata (SILVA et al., 1999). No entanto, apesar de a ausência de luz não ter sido fator limitante para a germinação, constatou-se que, para Livistona chinensis, a germinabilidade foi maior na luz do que no escuro, principalmente no parâmetro índice de velocidade de germinação.

Foi possível observar que o maior número de sementes germinou entre o sétimo e o décimo sétimo dias, com comportamento semelhante nos dois regimes de luz (figura $1 \mathrm{e}$ 2). Verificou-se que a germinação continuou elevada até próximo do vigésimo quinto dia, a partir do qual, para a maioria das temperaturas, não houve mais germinação, tendendo à estabilização do processo germinativo. É interessante observar que, à temperatura de $20^{\circ} \mathrm{C}$, em que foi constatado o menor IVG nos dois regimes de luz, a germinação continuou após os 25 dias, em quantidade, ao contrário do que foi visto a outras temperaturas, em que não houve mais germinação. A partir disso, pode-se concluir que a menor temperatura utilizada influenciou o metabolismo das sementes e seu desenvolvimento inicial. Isso porque a temperatura em que ocorre a germinação é um dos fatores essenciais no processo germi- nativo total e na velocidade de germinação.

Conforme BEWLEY \& BLACK (1985), a temperatura afeta tanto a capacidade como a velocidade de germinação, por influenciar a velocidade de absorção de água e também as relações bioquímicas que determinam todo o processo germinativo, uma sequência extremamente complexa, nas quais substâncias de reserva armazenadas são degradadas, transportadas e ressintetizadas no eixo embrionário. As sementes têm capacidade de germinar dentro de uma determinada faixa de temperatura, específica para cada espécie, mas o tempo necessário para se obter a porcentagem máxima de germinação é dependente da temperatura. De acordo com CARVALHO \& NAKAGAWA (2000), temperaturas inferiores ou superiores à ótima tendem a reduzir a velocidade do processo germinativo, expondo as plântulas por maior período a fatores adversos, o que pode levar à redução no total de germinação. A elevação da temperatura provoca redução da viscosidade e aumento da energia cinética da água, beneficiando a embebição e a velocidade das reações componentes do metabolismo (MARCOS FILHO, 2005). A redução gradativa da temperatura provoca decréscimo acentuado da velocidade de germinação, devido a seus efeitos sobre o tempo de embebição e de mobilização de reservas. 


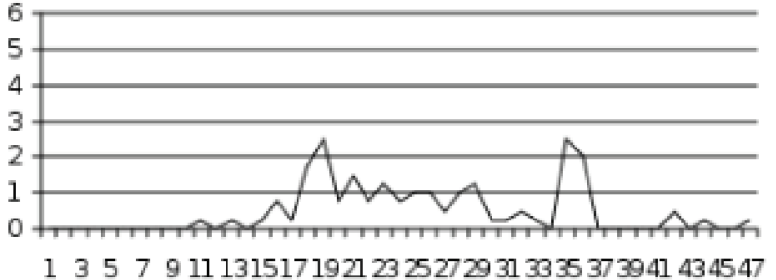

(A)

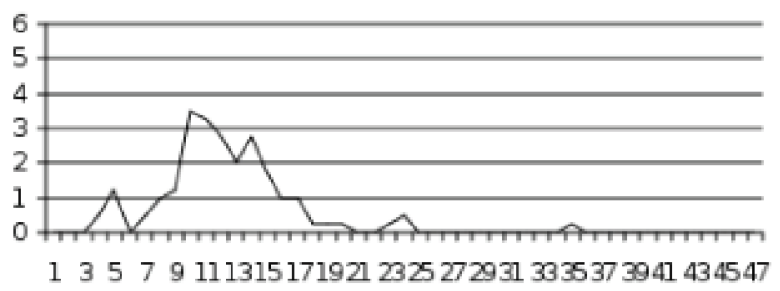

(C)

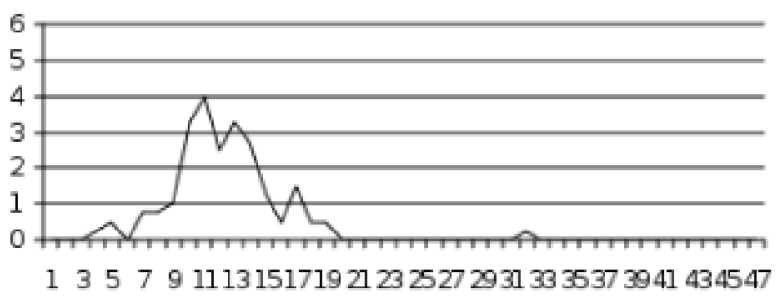

(E)

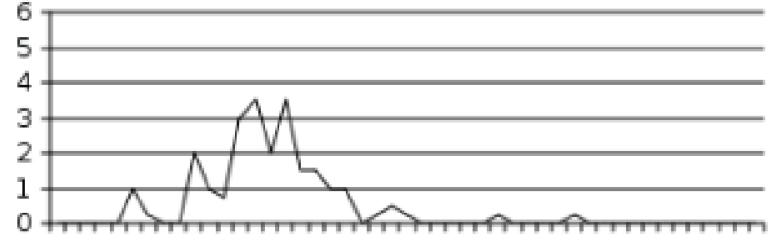

1357911131517192123252729313335373941434547

(B)

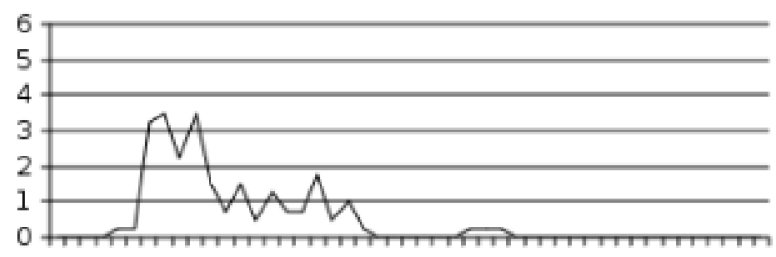

1357911131517192123252729313935373941434547

(D)

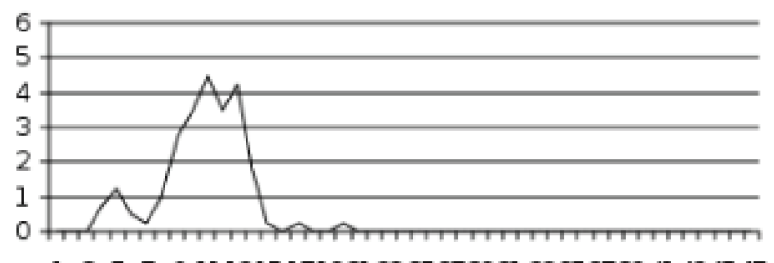

(F)

Figura 2. Número de sementes germinadas (eixo $\mathrm{Y}$ ) por dia (eixo $\mathrm{X}$ ) nas temperaturas de $20^{\circ} \mathrm{C}(\mathrm{A}), 25^{\circ} \mathrm{C}(\mathrm{B}), 30^{\circ} \mathrm{C}(\mathrm{C})$, $35^{\circ} \mathrm{C}(\mathrm{D}), 20-30^{\circ} \mathrm{C}(\mathrm{E}), 25-35^{\circ} \mathrm{C}(\mathrm{F})$ em escuro contínuo.

Figure 2. Number of germinated seeds (Y axis) per day (X axis) at temperatures of $20^{\circ} \mathrm{C}(\mathrm{A}), 25^{\circ} \mathrm{C}(\mathrm{B}), 30^{\circ} \mathrm{C}(\mathrm{C}), 35^{\circ} \mathrm{C}$ (D), $20-30^{\circ} \mathrm{C}(E), 25-35^{\circ} \mathrm{C}(F)$ in continuous dark.

Isso foi observado principalmente na porcentagem e índice de velocidade de germinação de sementes de Pameira-leque-da-China a $20^{\circ} \mathrm{C}$. Resultados semelhantes foram constatados para jerivá (PIVETTA et al., 2005b) e tamareira-anã (IOSSI et al., 2003) à temperatura de $30^{\circ} \mathrm{C}$. Para Thrinax parviflora (PIVETTA et al., 2005a), com exceção de $20^{\circ} \mathrm{C}$, as demais temperaturas proporcionaram melhores resultados e não diferiram entre si $\left(25,30,35^{\circ} \mathrm{C}\right.$ e $20-30 \mathrm{e}$ $25-35^{\circ} \mathrm{C}$ ). A temperatura alternada de $25-35^{\circ} \mathrm{C}$ proporcionou maior índice de velocidade de germinação para Livistona rotundifolia, bem como para a espécie Livistona chinensi (VIANA, 2003). A alternância de temperatura, na faixa entre 20 a $35^{\circ} \mathrm{C}$ pode proporcionar melhores condições de germinação para sementes de algumas espécies tropicais por ativar mecanismos enzimáticos que funcionam em diferentes temperaturas (VÁZQUEZ-YANES \& OROZCOSEGOVIA 1987), provavelmente indicando uma adaptação às flutuações naturais do ambiente (BORGES \& RENA, 1993). Para as sementes de palmeira-leque-da-China, entretanto, a alternância da temperatura não favoreceu a germinação, mas foi a responsável pelo aumento da velocidade de germinação, assim como constatado por GOMES \& BRUNO (1992) para Bixa orellana (L.), e por CASTELLANI \& AGUIAR (1998) para Trema micrantha (L.) Blume.

As sementes de Livistona chinensis (Jack.) R. Br. ex. Mart. apresentam alta porcentagem de germinação, inde- pendentemente da temperatura e do regime de luz, e maior IVG nas temperaturas alternadas de $25-35^{\circ} \mathrm{C}$, com regime de 8 horas de luz, sendo estas as condições recomendadas para a condução do teste de germinação.

\section{AGRADECIMENTOS}

Os autores gostariam de agradecer à CAPES pela concessão da bolsa de estudo, e aos funcionários do Departamento de Produção Vegetal pela ajuda na realização deste trabalho.

\section{REFERÊNCIAS}

ANDRADE, A.C.S. Efeito da luz e da temperatura na germinação de Leandra breviflora Cogn., Tibouchina benthamiana Cogn., Tibouchina grandifolia Cogn. E Tibouchina moricandia (DC.) Baill. (Melastomataceae). Revista Brasileira de Sementes, v.17, p.29-35, 1995.

BANZATTO, D. A.; KRONKA, S. N. Experimentação agrícola. 4.ed. Jaboticabal: Funep, 2006. 237p.

BEWLEY, J. D.; BLACK, M. Seeds: physiology of development and germination. New York: Plenum Press, 1996. $445 \mathrm{p}$. 
BORGES, E. E. L.; RENA, A. B. Germinação de Sementes. In: AGUIAR, I. B.; PIÑA-RODRIGUES, F. C. M.; FIGLIOLIA, M. B. Sementes florestais tropicais. Brasília: Abrates, 1993. p.83-135.

BORGHETTI, F.; FERREIRA, A.G. Interpretação de resultados de germinação. In BORGHETTI, F.; FERREIRA, A.G. (orgs.) Germinação: do básico ao aplicado. Porto Alegre: ArtMed, p.209-222. 2004.

BRASIL. Ministério da Agricultura e Reforma Agrária. Regras para análise de sementes - RAS. Brasília: Secretaria Nacional de Defesa Agropecuária, 1992. 365p.

BROSCHAT, T. K. Palm seed propagation. Acta Horticulturae, Wageningen, v.360, p.141-147, 1994.

BRYANT, J.A. Fisiologia da semente. São Paulo: EPU, 1985.

CARPENTER, W. J. Temperature affects seed germination of four Florida palm species. HortScience, Alexandria, v.23, p.336-337, 1988.

CARVALHO, N. M.; NAKAGAWA, J. Sementes: ciência, tecnologia e produção. 4.ed. Jaboticabal: Funep, 2000. $588 \mathrm{p}$.

CASTELLANI, E.D.; AGUIAR, I.B. Condições preliminares para a germinação de sementes de candiúba (Trema micrantha (L.) Blume). Revista Brasileira de Engenharia Agrícola e Ambiental v 2, n. 1, p. 80-83. janeiro-abril,1998.

CASTRO, R. D.; HILHORST, H. W. M. Embebição e reativação do metabolismo. In: FERREIRA, A. G.; BORGHETTI, F. (orgs.). Germinação: do básico ao aplicado. Porto Alegre: ArtMed, 2004. p.149-162.

CHATTY, Y.; TISSAOUI, T. Effect of temperature on germination of ornamental palm trees in Tunisia. Acta Horticulturae, Wageningen, v.486, p.165-167, 1999.

ELLIS, R. H.; HONG, T. D.; ROBERTS, E. H. Handbook of seed technology for genebanks. v.2. Compendium of specific germination information and test recommendations. Rome: Board for Plant Genetic Resources (IBPGR), 1985. 667p.

GILMAN, E. F.; WATSON, D. G. Livistona chinensis: Chinese Fan Palm. Florida: Cooperative Extension Service, 1993. 4p. (Fact Sheet ST, 365).

GOMES, S.M.S.; BRUNO, R.L.A. Influência da temperatura e substratos na germinação de sementes de urucum (Bixa orellana L.). Revista Brasileira de Sementes, v.14, p.47-50, 1992.

KALIL FILHO, A. N.; REZENDE, M. D. V. Melhoramento de palmáceas. In: WORKSHOP SOBRE MELHORA-
MENTO DE ESPÉCIES FLORESTAIS E PALMÁCEAS NO BRASIL, 1., 2001, Colombo. Série Documentos. Colombo: Embrapa Florestas, 2001, n.62, p. 95-114.

LORENZI, H.; SOUZA, H. M.; COSTA. J. T. M.; CERQUEIRA, L. S. C.; FERREIRA, E. Palmeiras brasileiras e exóticas cultivadas. Nova Odessa: Plantarum, 2004. 416p.

MAGUIRE, J. D. Speed of germination - aid in selection and evaluation for seedling emergence and vigor. Crop Science, Madison, v.2, n.2, p.176-177, 1962.

MARCOS FILHO, J. Fisiologia de sementes de plantas cultivadas. Piracicaba: FEALQ, 2005. 495p.

MEEROW, A. W. Betrocks guide to landscape palms. Cooper City: Betrocks Information Systems, 1991a. 153p.

MEEROW, A. W. Palm seed germination. Florida: Cooperative Extension Service, 1991b. 10p. (Bulletin, 274).

MELO, J. R. V. Maturação, germinação e armazenamento de sementes de piaçaveira (Attalea funifera Mart.). Botucatu, 2001. 115f. Tese (Doutorado em Agricultura) - Faculdade de Ciências Agronômicas, Universidade Estadual Paulista.

OLIVEIRA, E. C.; PIÑA-RODRIGUES, F. C. M.; FIGLIOLIA, M. B. Propostas para a padronização de metodologias em análise de sementes florestais. Revista Brasileira de Sementes, Brasília, v.11, n.1,2,3. p.1-25. 1989.

PENARIOL, A. P. Efeito da temperatura e do estádio de maturação na germinação de sementes de Roystonea regia (Kunth) O.F. Cook (Arecaceae)., Jaboticabal, 2005. 32f. Monografia (Trabalho de Graduação em Agronomia) - Faculdade de Ciências Agrárias e Veterinárias, Universidade Estadual Paulista.

PINTO, M. F. Germinação acelerada de sementes de palmeira. Agronomia Angolana, Luanda, v.31, n.1, p.1-17, 1971

PIVETTA, K. F. L.; PAULA, R. C.; CINTRA, G. S.; PEDRINHO, D. R.; CASALI, L. P.; PIZETTA, P. U. C.; PIMENTA, R. S. Effects of temperature on seed germination of Queen Palm Syagrus romanzoffiana (Cham.) Glassman. (Arecaceae). Acta Horticulturae, Leuven, v.683, p.379$381,2005 b$.

PIVETTA, K. F. L.; CASALI, L. P.; CINTRA, G. S.; PEDRINHO, D. R.; PIZETTA, P. U. C.; PIMENTA, R. S.; PENARIOL, A. P.; MATTIUZ, C. F. M. Efeito da temperatura e do armazenamento na germinação de sementes de Thrinax parviflora swartz. (Arecaceae). Científica, Jaboticabal, v.33, n.2, p.178-184, 2005a.

REES, W. A. Germination of palm seeds using a method developed for the oil palm. Principes, Lawrence, v.7, n.1, p.27-30, 1963. 
SILVA, M. A. S.; Castellani, E. D.; Demattê, M. E. S. P. Effect of fruit maturation stage and light on seed germination. Acta Horticulturae, v.486, p.229-234, 1999.

VÁZQUEZ-YANES, C. \& OROZCO-SEGOVIA, A. Fisiología ecológica de semillas en la Estación de Biologia Tropical "Los Tuxtlas", Veracruz, México. Revista de Biologia Tropical, v.35, p.85-96, 1987.

VÁZQUEZ-YANES, C. \& OROZCO-SEGOVIA, A. Ecological significance of light controlled seed germination in two contrasting tropical habitats. Oecologia, v.83 p. 171175, 1990.

VÁZQUEZ-YANES, C. \& OROZCO-SEGOVIA, A. 1991. Seed viability, longevity and dormancy in a tropical rain forest. In Anais do II Simpósio Brasileiro sobre Tecnologia de Sementes Florestais (FIGLIOOLIA, M.B., coord.). São Paulo: Instituto Florestal, p.175-196.

VIANA, F. A. P. Estudos sobre germinação e morfo-anatomia do diásporo e da plântula de Livistona rotundifolia
(Lam.) Mart. (Arecaceae). Jaboticabal, 2003. 76f. Dissertação (Mestrado em Produção e Tecnologia de Sementes) - Faculdade de Ciências Agrárias e Veterinárias, Universidade Estadual Paulista.

VIDAVER, W. 1980. Light and seed germination. In The physiology and biochemistry of seed dormancy and germination (KHAN, A.A., ed.). New York: North-Holland Publishing Company, p.181-192.

VILLALOBOS, R.; HERRERA, J. Seed germination in pejibaye palm (Bactris gasipaes): effect of temperature and substrate. Agronomía Costarricense, San Jose, v.15, n.1/2, p.57-62, 1991.

YOCUM, H.G. A method for germinating palm seeds. Principes, v.5, n.1, p.31-32, 1961.

ZAIDAN, L. B. P.; BARBEDO, C. J. Quebra de dormência em sementes. In: FERREIRA, A. G.; BORGHETTI, F. (Ed.). Germinação: do básico ao aplicado. Porto Alegre: ArtMed, 2004. p.135-146. 
